$\begin{array}{lrlll}\text { November } & 8, & 2018 & 23: 3 & \text { WSPC/INSTRUCTION }\end{array}$ EncripletasvF-IJMPC

International Journal of Modern Physics C

(C) World Scientific Publishing Company

\title{
IMPROVEMENT AND ANALYSIS OF A PSEUDO RANDOM BIT GENERATOR BY MEANS OF CELLULAR AUTOMATA
}

\author{
J. S. MURGUÍA * \\ UASLP, Universidad Autónoma de San Luis Potosí \\ Álvaro Obregón 64, 78000 San Luis Potosí, S.L.P., México \\ IPICyT, Instituto Potosino de Investigacion Cientifica y Tecnologica \\ Apartado Postal 3-74 Tangamanga, 78231 San Luis Potosí, México \\ ondeleto@uaslp.mx \\ M. MEJÍA CARLOS \\ Instituto en Investigación en Comunicación Óptica, UASLP \\ Álvaro Obregón 64, 78000 San Luis Potosí, S.L.P., México \\ mmejia@cactus.iico.uaslp.mx \\ H.C. ROSU \\ IPICyT, Instituto Potosino de Investigacion Cientifica y Tecnologica \\ Apartado Postal 3-74 Tangamanga, 78231 San Luis Potosí, México \\ hcr@ipicyt.edu.mx

\section{G. FLORES-ERANA} \\ Instituto en Investigación en Comunicación Óptica, UASLP \\ Álvaro Obregón 64, 78000 San Luis Potosí, S.L.P., México \\ gustavo.flores.erana@gmail.com
}

Received Day Month Year Revised Day Month Year

\begin{abstract}
In this paper, we implement a revised pseudo random bit generator based on a rule-90 cellular automaton. For this purpose, we introduce a sequence matrix $H_{N}$ with the aim of calculating the pseudo random sequences of $N$ bits employing the algorithm related to the automaton backward evolution. In addition, a multifractal structure of the matrix $H_{N}$ is revealed and quantified according to the multifractal formalism. The latter analysis could help to disentangle what kind of automaton rule is used in the randomization process and therefore it could be useful in cryptanalysis. Moreover, the conditions are found under which this pseudo random generator passes all the statistical tests provided by the National Institute of Standards and Technology (NIST).
\end{abstract}

Keywords: Cellular automata, pseudo-random generator; multifractal spectrum.

PACS numbers.: 05.40.-a., 05.45.-a., 05.45.Tp

${ }^{*}$ Corresponding author 
November EncripletasvF-IJMPC

\section{Introduction}

Random numbers constitute one of the main ingredients in a great number of applications such as cryptography, simulation, games, sampling, and so on. Cellular automata (CA) offer a number of advantages over other methods as random number generators (RNG), such as algorithmic simplicity and easy hardware implementation. In fact, CA are highly parallel and distributed systems which are able to perform complex computations.

Over the last years, researchers have applied cellular automata in pseudo-random number generation (PRNG) 12/3/4|5. These PRNGs must possess a number of properties if they are to be used for cryptographic application. The most important from this point of view are good results on standard statistical tests of randomness, computational efficiency, a long period, and reproducibility of the sequence 6 . In many encryption systems, PRNGs are used to get keys, which are generated from an initial seed, and they are reproducible if the same seed is used.

Wolfram was the first to apply the one-dimensional elementary cellular automata (ECA) to obtain PRNGs 7. He considered only the use of the rule 30 in one dimension with radius 1 . Other authors have used non-uniform ECA $5[8 \mid 9$, where they have found that the quality of the latter PRNGs was better than the quality of Wolfram's system.

Despite these works demonstrate an improvement in the quality of PRNGs, this study is devoted to an extension of the analysis of the evolutionary technique for getting PRNGs based on a uniform ECA with rule 902. Namely, we consider a modification of the generator producing PRGNs. The initial proposal has been never implemented and studied in terms of the sequence matrix $H_{N}$, which is used here to generate recursively the pseudo random sequences. The time series of the row sums of the matrix $H_{N}$ are also analyzed within the multifractal formalism because they could be a possible useful feature for cryptanalysis of these types of PRNGs. In order to check the quality of this ECA-PRNG, the generated sequences are evaluated statistically by the NIST suite. The generated sequences, in length terms, pass all the statistical tests proposed by NIST. Our results suggest that this generator in its two versions that we discuss in the following fit naturally in the present digital communication systems and achieve high levels of performance.

\section{Elementary Cellular Automata}

The ECA can be considered as discrete dynamical systems that evolve in discrete time steps. The state space of a CA of size $N$ is the set $\Omega=\mathbb{Z}_{k}^{N}$ of all sequences of $N$ cells that take values from $\mathbb{Z}_{k}=\{0,1, \ldots, k-1\}$, where its evolution is defined by the repeated iteration of an evolution operator $\mathcal{A}: \mathbb{Z}_{k}^{N} \rightarrow \mathbb{Z}_{k}^{N}$. In this paper, we consider $k=2$ where $\mathbb{Z}$ is the set of integers. An automaton state $\underline{x} \in \mathbb{Z}_{k}^{\mathbb{Z}}$ has coordinates $(\underline{x})_{i}=x_{i} \in \mathbb{Z}_{2}$ with $i \in \mathbb{Z}$, and the automaton state at time $t \geq 0$ is denoted by $\underline{x}^{t} \in \mathbb{Z}_{2}^{\mathbb{Z}}$ and its evolution is defined iteratively by the rule $\underline{x}^{t+1}=A\left(\underline{x}^{t}\right)$. Starting from the initial state $\underline{x}^{0}$, the automaton generates the forward space-time 
pattern $\mathbf{x} \in \mathbb{Z}_{2}^{\mathbb{Z} \times \mathbb{N}}$ with state $(\mathbf{x})^{t}=\underline{x}^{t}=A^{t}\left(\underline{x}^{0}\right)$ reached at from $\underline{x}^{0}$ after $t \in \mathbb{N}$ time steps. $\mathbb{N}$ denotes the set of nonnegative integers.

One can see that the time, space, and states of this system take only discrete values. The ECA considered evolves according to the local rule $x_{i}^{t+1}=$ $\mathcal{A}_{L}\left(x_{i-1}^{t}, x_{i}^{t}, x_{i+1}^{t}\right)=\left[x_{i-1}^{t}+x_{i}^{t}\right] \bmod 2$, which corresponds to the rule 90 . The following is the lookup table of rule 90 .

\begin{tabular}{|l|c|c|c|c|c|c|c|c|}
\hline Number & 7 & 6 & 5 & 4 & 3 & 2 & 1 & 0 \\
\hline Neighborhood & 111 & 110 & 101 & 100 & 011 & 010 & 001 & 000 \\
\hline Rule result & 0 & 1 & 0 & 1 & 1 & 0 & 1 & 0 \\
\hline
\end{tabular}

The third row shows the future state of the cell if itself and its neighbors are in the arrangement shown above in the second row. In fact, a rule is numbered by the unsigned decimal equivalent of the binary expression in the third row. When the same rule is applied to update cells of ECA, such ECA are called uniform ECA; otherwise the ECA are called non-uniform or hybrids. It is important to observe that the evolution rules of ECA are determined by two main factors, the rule and the initial conditions.

\section{Pseudo Random Sequences Generator}

In Ref. 2, Mejía and Urías presented an ergodic and mixing transformation of binary sequences in terms of a cellular automaton, which is the main element of a pseudorandom generator number (PRGN). To implement numerically the PRGN in its basic form, we follow their algorithm, which is shown in Fig. 1. At first, the key generator requires two seeds, $\mathbf{x}=\mathbf{x}_{0}^{k+1}$, of $N$ bits, and $\mathbf{y}=\mathbf{x}_{0}^{k}$, of $(N+1)$ bits, which are the input of function $\mathbf{t}=h(\mathbf{x}, \mathbf{y})$. The seeds are $\mathbf{x}=\left\{x_{1}, x_{2}, x_{3}, \ldots, x_{N}\right\}$ and $\mathbf{y}=\left\{y_{1}, y_{2}, y_{3}, \ldots, y_{N+1}\right\}$, and the first number generated of $N$ bits is the sequence output of function $h, \mathbf{t}=x_{0}^{1}=\left\{t_{1}, t_{2}, t_{3}, \ldots, t_{N}\right\}$. Now this sequence is feeding back to the input, which becomes the next value of $\mathbf{x}$, and the previous value of $\mathbf{x}$ becomes the initial bits of the new $\mathbf{y}$, where the missing bit is the least significant bit (LSB) of the previous $\mathbf{y}$, which becomes the most significant bit (MSB) of this sequence, and the same procedure is iterated repeatedly.

The previous description to compute the function $\mathbf{t}=h(\mathbf{x}, \mathbf{y})$ requires that the cellular automaton runs backwards in time as is depicted in Fig. 2, The symbol of a circled + represents a XOR gate and the connectivity of gates follows the automaton rule. However, this way to compute the pseudo-random sequences is not efficient since it requires the application of the local rule of the automaton at all points in a lattice of the order of $N^{2}$, where $N$ is the number of bits considered in the generation process. Fig. 3 (a) shows a complete evolution in the lattice of the generator considered with $N=31$ bits, where the two left columns comprise the seed $(\mathbf{x}, \mathbf{y})$, the top row is the resulting pseudo random key sequence $\mathbf{t}$, and the intermediate elementary computations are the rest. A clear dot represents a bit 
November 8

value of 1 , whereas a dark dot corresponds to a bit value of 0 .

To overcome this, Mejía and Urías 2 formulated an efficient algorithm that gets rid of the intermediate variables and produces boolean expressions for the coordinates of the output sequence $\mathbf{t}=h(\mathbf{x}, \mathbf{y})$ in terms of the input $(\mathbf{x}, \mathbf{y})$. This algorithm offers a boolean representation of $h$, without intermediate steps, in terms of some "triangles" in the underlying lattice. The pattern of triangles is observed in Fig. 3(a). In fact, it is well known that the evolution of rule 90 has the appearance of a Sierpinski triangle when responding to an impulse, i.e., when the first row is all 0 s with a 1 in the center.

We introduce now the sequence matrix $H_{N}$, which computes the pseudo-random sequences of $N$ bits. This matrix has dimensions of $(2 N+1) \times(2 N+1)$ and is formed by the matrices $H_{N_{t}}$ and $H_{N_{b}}$, which constitute the top and bottom parts of $H_{N}$, that is, $H_{N}=\left(H_{N_{t}} ; H_{N_{b}}\right)$. The matrix $H_{N_{t}}$ has dimensions of $N \times(2 N+1)$ elements and it is generated initially from vectors $\mathbf{v}=\left[v_{1}, 0, \ldots, 0, v_{N+2}, \ldots, 0\right]$ and $\mathbf{w}=$ $\left[0, w_{2}, 0, \ldots, w_{N+1}, 0, w_{N+3}, \ldots, 0\right]$, where the components $v_{1}, v_{N+2}, w_{2}, w_{N+1}$ and $w_{N+3}$ have a value of 1 , and $N$ is the number of bits, i. e., $\mathbf{v}$ and $\mathbf{w}$ are vectors with $(2 N+1)$ elements. The vectors $\mathbf{v}$ and $\mathbf{w}$ constitute the two first rows of the matrix $H_{N_{t}}$ and the $(N-2)$ rows are generated applying an addition modulo 2 operation of the two previous rows, with the elements of the previous row shifted to the right by one position. The matrix $H_{N_{t}}$, of dimensions $(N+1) \times(2 N+1)$, has a simpler form, an identity matrix in the first $(N+1)$ columns and zeros in the rest. For instance, for $N=3$ we have that the top and bottom matrices are

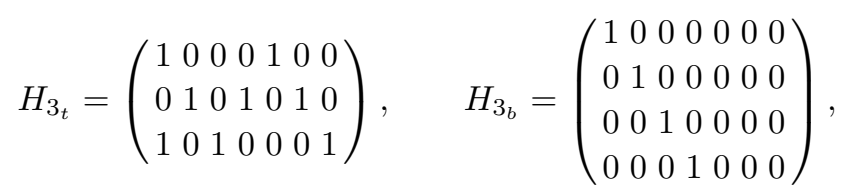

then the matrix $H_{3}$ is

$$
H_{3}=\left(\begin{array}{l}
H_{3_{t}} \\
H_{3_{b}}
\end{array}\right)=\left(\begin{array}{lllllll}
1 & 0 & 0 & 0 & 1 & 0 & 0 \\
0 & 1 & 0 & 1 & 0 & 1 & 0 \\
1 & 0 & 1 & 0 & 0 & 0 & 1 \\
1 & 0 & 0 & 0 & 0 & 0 & 0 \\
0 & 1 & 0 & 0 & 0 & 0 & 0 \\
0 & 0 & 1 & 0 & 0 & 0 & 0 \\
0 & 0 & 0 & 1 & 0 & 0 & 0
\end{array}\right)
$$

Notice that $H_{N_{t}}$ computes the pseudo random key sequence, whereas $H_{N_{b}}$ the feedback sequence. Therefore, once selected the number $N$ of bits of sequences, we can generate the pseudo random sequences of $N$ bits with the help of the matrix $H_{N}$

$$
\mathbf{U}_{k+1}=H_{N} \mathbf{U}_{k}, \quad k=1,2, \ldots
$$


November EncripletasvF-IJMPC

where $\mathbf{U}_{k}=\left[\begin{array}{ll}\mathbf{x} & \mathbf{y}\end{array}\right]^{T}$ corresponds to the first inputs of function $h$, and $\mathbf{U}_{k+1}$ is composed by the next inputs of $h$; note that $\mathbf{U}_{k+1}$ is formed by the generated pseudo random key and the feedback sequence.

\subsection{Modified generator}

As was pointed out in Ref. 2, a generating scheme consisting of three coupled transformations $h$ is proposed to attain an asymptotically unpredictable generator under a random search attack. This proposal is shown in Fig. 4 and it is explained briefly. Inside the new generator two copies of the basic transformation $h$ are iterated autonomously from their initial words generating two sequences, $\left\{p^{k}\right\}_{k \geq 0}$ and $\left\{q^{k}\right\}_{k \geq 0}$. The third copy, called the $x$-map, is iterated in a slightly different manner, the function $h$ in the $x$-map is driven by the autonomous $p$-map and $q$-map according to $x^{k}=h\left(p^{k}, q^{k}\right)$. The three maps generate pseudo random sequences, but just the $x$ sequence is released. In order to prevent predictability, the first two words are generated, used and destroyed inside this key generator, therefore they are not available externally. Since the sequences $p^{k}$ and $q^{k}$ have a length of $N$ bits, and the required inputs of the $h$ transformation must be one of $N$ bits and other of $(N+1)$ bits, the missing bit is obtained applying an addition modulo 2 operation between the two respective LSB's that become the MSB's of their respective previous inputs of maps. Of course, there exists different manners to generate this missing bit, but we consider this way. The above scheme has been just proposed, but it has not been implemented and studied in terms of the matrix sequence. The new pseudo-random keys are computed as

$$
\mathbf{X}_{N}=H_{N_{t}} \mathbf{V}_{N}
$$

where $\mathbf{X}_{N}=\left\{x_{1}, x_{2}, \ldots, x_{N}\right\}^{T}, H_{N_{t}}$ is the top matrix of $H_{N}$, and $\mathbf{V}_{N}=$ $\left\{p_{1}, \ldots, p_{N}, q_{1}, \ldots, q_{N+1}\right\}^{T}$. For example, considering $N=3$, we have

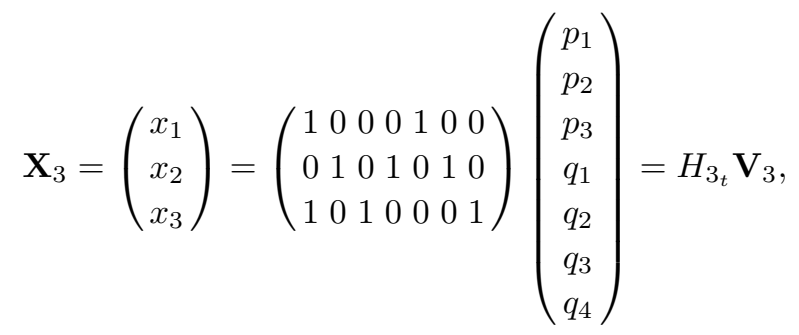

where we calculate $p_{i}$ and $q_{i}$ as it was explained above. Fig. [3 (b) shows the complete evolution of this modified generator for 31 bits, with the $p$-map on the left side, the $q$-map on the middle, and the $x$-map on the right side. 
\begin{tabular}{|lllll} 
November & 8, & 2018 & $23: 3$ & WSPC/INSTRUCTION
\end{tabular} EncripletasvF-IJMPC

\subsection{Multifractal properties of the matrix $\mathrm{H}_{N}$}

Since the evolution of the sequence matrix $H_{N}$ is based on the evolution of the CA rule 90 , the structure of the patterns of bits of the latter are directly reflected in the structure of the entries of $H_{N}$. There is recent literature on the multifractal properties of cellular automata for some set of rules, see 10|11/12. In Ref. 13, we used the technique of detrended fluctuation analysis based on the discrete wavelet transform (WMF-DFA) to quantify the intrinsic multifractal behavior of the ECAs for rules 90,105 , and 150 .

Here, in the same spirit as in Ref. 13, we analyze the sum of ones in the sequences of the rows of the matrix $H_{N}$ with the db- 4 wavelet, a wavelet function that belongs to the Daubechies family 14 .

The results for two row sums, $H_{1023}$ and $H_{2047}$, are illustrated in Figs. 556 We confirm the multifractality of both time series since we get a $\tau$ spectrum with two slopes in both cases. The strength of the multifractality is roughly measured with the width $\Delta \alpha=\alpha_{\max }-\alpha_{\min }$ of the parabolic singularity spectrum $f(\alpha)$ on the $\alpha$ axis. For the case of $H_{1023}$ the width $\Delta \alpha_{H_{1023}}=1.16-0.212=0.948$, and the most "frequent" singularity occurs at $\alpha_{\mathrm{mf}_{\mathrm{H}_{1023}}}=0.694$, whereas for $H_{2047}$,

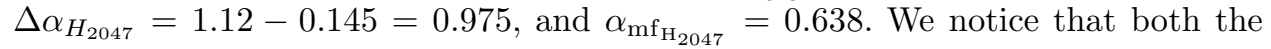
strongest singularity, $\alpha_{\min }$, and the weakest singularity, $\alpha_{\max }$, are very similar as well as the most "frequent" singularity. These results are in a good agreement with those obtained in Ref. 13 for the rule 90, although the spectra of the top matrix present a slight shifting to the right. In fact, this behavior is more evident in the row signals of $H_{N}$ (Figs. 可6 (c)), where their corresponding spectra (dotted points) are shown in Figs. 5.6 (f).

\section{Statistical Tests}

There are several options available for analyzing the randomness of the pseudo random bit generators. The four most popular options according to Ref. 15 are the following: NIST suite of statistical tests, the DIEHARD suite of statistical tests, the Crypt-XS suite of statistical tests and the Donald Knuth's statistical test set.

In this paper, we consider the NIST suite to analyze the generated pseudo random sequence keys. The main reason is that this suite has several appealing properties 16/17. For instance, it is uniform, it is composed by a number of well known tests and, for all of them, an exhaustive mathematical treatment is available. In addition, the source code of all tests in the suite is public available and is regularly updated 16. In fact, in Ref. 16 is mentioned that the NIST suite may be useful as a first step in determining whether or not a generator is suitable for a particular cryptographic application.

The NIST suite is a statistical package consisting of 15 tests that were developed to test the randomness of (arbitrarily long) binary sequences produced by either hardware or software based cryptographic random or pseudo-random number generators. These tests focus on a variety of different types of non-randomness that 
November 8, $2018 \quad 23: 3 \quad$ WSPC/INSTRUCTION $\quad$ FILE EncripletasvF-IJMPC

could exist in a sequence. Some tests are decomposable into a variety of subtests, and the 15 tests are listed in Table 1.

Table 1. List of NIST Statistical Tests.

\begin{tabular}{cc}
\hline Number & Test name \\
\hline 1 & The Frequency (Monobit) Test \\
2 & Frequency Test within a Block \\
3 & The Runs Test \\
4 & Tests for the Longest-Run-of-Ones in a Block \\
5 & The Binary Matrix Rank Test \\
6 & The Discrete Fourier Transform (Spectral) Test \\
7 & The Non-overlapping Template Matching Test \\
8 & The Overlapping Template Matching Test \\
9 & Maurer's "Universal Statistical" Test \\
10 & The Linear Complexity Test \\
11 & The Serial Test \\
12 & The Approximate Entropy Test \\
13 & The Cumulative Sums (Cusums) Test \\
14 & The Random Excursions Test \\
15 & The Random Excursions Variant Test \\
\hline
\end{tabular}

For each statistical test, a set of $P$-values (corresponding to the set of sequences) is produced. For a fixed significance level $\alpha$, a certain percentage of $P$ - values are expected to pass/fail the tests. For example, if the significance level is chosen to be 0.01 (i.e., $\alpha=0.01$ ), then about $1 \%$ of the sequences are expected to fail. A sequence passes a statistical test whenever the $P-$ value $\geq \alpha$ and fails otherwise. For each statistical test, the proportion of sequences that pass is computed and analyzed accordingly. It is not sufficient to look solely at the acceptance rates and declare that the generator be random if they seem fine. If the test sequences are truly random, the $P$-values calculated are expected to appear uniform in $[0,1]$.

For the interpretation of test results, NIST has adopted two approaches, (1) the examination of the proportion of sequences that pass a statistical test and (2) the distribution of $P$-values to check for uniformity.

- Proportions of the sequence passing the tests: For each test is computed the proportion of sequences that passes the tests. First, the range of acceptable proportions is determined using the confidence interval, which is defined as

$$
\hat{p} \pm 3 \sqrt{\frac{\hat{p}(1-\hat{p})}{m}},
$$

where $\hat{p}=1-\alpha$, and $m$ is the sample size. If the proportion falls outside of this interval, then there is evidence that the data is non-random.

- Uniform Distribution of $P$-values: The distribution of $\mathrm{P}$-values is examined to ensure uniformity. This may be visually illustrated using a histogram. It may be 
November EncripletasvF-IJMPC

also computed by means of a chi-square test $\left(\chi^{2}\right)$, and the determination of a $P$ - value of the $P$-values. The computation is as follows:

$$
\chi^{2}=\sum_{i=1}^{10} \frac{\left(f_{i}-\frac{m}{10}\right)^{2}}{\frac{m}{10}},
$$

where $f_{i}$ is the number of $P$-values in the sub-interval $i$ and $m$ is the size of the sample, which is $m=100$ for the present analysis. A $P$-value is calculated such that $P-$ value $_{T}=\operatorname{igamc}\left(9 / 2, \chi^{2} / 2\right)$, where igamc $(n, x)$ is the incomplete gamma function. If $P-$ value $_{T} \geq 0.0001$, then the sequences can be considered to be uniformly distributed.

\subsection{Results of the NIST statistical test suite}

For the present statistical test, the two analyses described above are applied and evaluated to determine if the generated sequences are random or not. We have considered $m=100$ samples of $10^{6}$ bit sequences, where each sequence has been generated from a randomly chosen seed, and the proportion must lie above $0.960150(\alpha=0.01)$ and $P-$ value $_{T} \geq 0.0001$. In order to investigate the performance of the generator, we analyze the generated pseudo-random sequences for $N=7, N=15$ and $N=31$ bits, considering one and three transformations.

\subsubsection{Case of $N=7$ bits}

In Figs. 7 7 8 are shown the results from the NIST testing for $N=7$ bits for one and three transformations, respectively. We can observe that in this case there is a poor performance; the generated pseudo random sequences just passes some tests and are not uniformly distributed.

\subsubsection{Case of $N=15$ bits}

Figs. 9.10 show the results for $N=15$ bits for one and three transformations, respectively. There is better performance that in the previous case. We can observe that using one transformation, the generator do not passes all tests, see Fig. 9. but it is uniformly distributed.

\subsubsection{Case of $N=31$ bits}

In the last case, Figs. 11 12 show the results for $N=31$ bits for one and three transformations, respectively. As we can see, all tests are passed using one and three transformations.

\section{Conclusions}

We have implemented and reviewed a pseudo-random number generator based on a rule-90 cellular automata. This generator in its basic form (using one transforma- 
November 8, $2018 \quad 23: 3 \quad$ WSPC/INSTRUCTION $\quad$ FILE EncripletasvF-IJMPC

tion), and its modified version (with three transformations) are analyzed by means of a sequence matrix $H_{N}$. The intrinsic multifractal properties of the sequence matrix in the two versions of the generator are discussed having in mind their possible usage in cryptanalysis. In addition, the quality of the generated pseudo random sequences are evaluated using the NIST statistical tests. According to these tests, this PRNG can generate high-quality random numbers using one or three transformations. It is worth noticing that the longer the length of the generated numbers the better is the quality of the random numbers we obtain. In other words, the generator will produce sequences of keys with a better quality as the size of keys is increased. The only case that fails to pass all the tests with one or three transformations is for $N=7$ bits. There are also some statistical problems for $N=15$ bits using one transformation. We were able to obtain random sequences of 15 bits, without repeating, of period length $2^{27}$ and $2^{31}$ using one and three transformations, respectively. These results are sufficient for many cryptographic applications.

\section{Acknowledgments}

G. Flores-Eraña is a doctoral fellow of CONACYT (México) in the program of "Ciencias Aplicadas" at IICO-UASLP.

\section{References}

1. J. Urías, E. Ugalde and G. Salazar, Chaos, 8, 819 (1998).

2. M. Mejía and J. Urías, Discrete and Continuous Dynamical Systems, 7, 115 (2001).

3. M. Mejía Carlos,, Ph. D. thesis, Universidad Autónoma de San Luis Potosí, SLP (2001).

4. M. Sipper and M. Tomassini, International Journal of Modern Physics C, 7, 181 (1996).

5. F. Seredynski, P. Bouvry and A. Y. Zomaya, Parallel Computing, 30, 753 (2004).

6. G. Zied, M. Mohsen, Z. Medien and T. Rached, International Journal of Computer Sciences and Engineering Systems, 2, 185 (2008).

7. S. Wolfram, Lecture Notes in Computer Science, 218, 429 (1986).

8. P. D. Hortensius, R. D. McLeod, D. M. Miller, and H. C. Card, IEEE Transactions on Computer, 38, 1466 (1989).

9. S. Nandi, B.K. Kar and P.P. Chaudhuri, IEEE Transactions on Computer, 43, 1346 (1994).

10. J. R. Sanchez, International Journal of Modern Physics C, 14, 491 (2003).

11. J.R. Sanchez and R. Alonso-Sanz, International Journal of Modern Physics C, 15, 1461 (2004).

12. J. Nagler and J.C. Claussen, Phys. Rev. E, 71, 067103 (2005).

13. J. S. Murguia, J. E. Perez-Terrazas, and H. C. Rosu, Europhysics Letters, 87, 28003 (2009).

14. S. Mallat, A Wavelet Tour of Signal Processing, (Academic Press, 2nd. Ed., 1999).

15. V. Patidar and K. K. Sud, Electronic Journal of Theoretical Physics, 6, 327 (2009).

16. A. Rukhin, J. Soto, J. Nechvatal, M. Smid, E. Barker, S. Leigh, M. Levenson, M. Vangel, D. Banks, A. Heckert, J. Dray and S. Vo, NIST Special Pub. 800-22 Rev. 1, http://csrc.nist.gov/rng/ (2008). 
$\begin{array}{lrlll}\text { November } & 8, & 2018 & 23: 3 & \text { WSPC/INSTRUCTION }\end{array}$ EncripletasvF-IJMPC

10 Murguía, Mejía Carlos, Rosu \& Flores-Eraña

17. C. Kenny, Random Number Generators: An Evaluation and Comparison of Random.org and Some Commonly Used Generatos, Trinity Collage Dublin, Management Sciencie and Information Systems Studies Project Report (2005). 
$\begin{array}{lllll}\text { November } & 8, & 2018 & 23: 3 & \text { WSPC/INSTRUCTION }\end{array}$ EncripletasvF-IJMPC

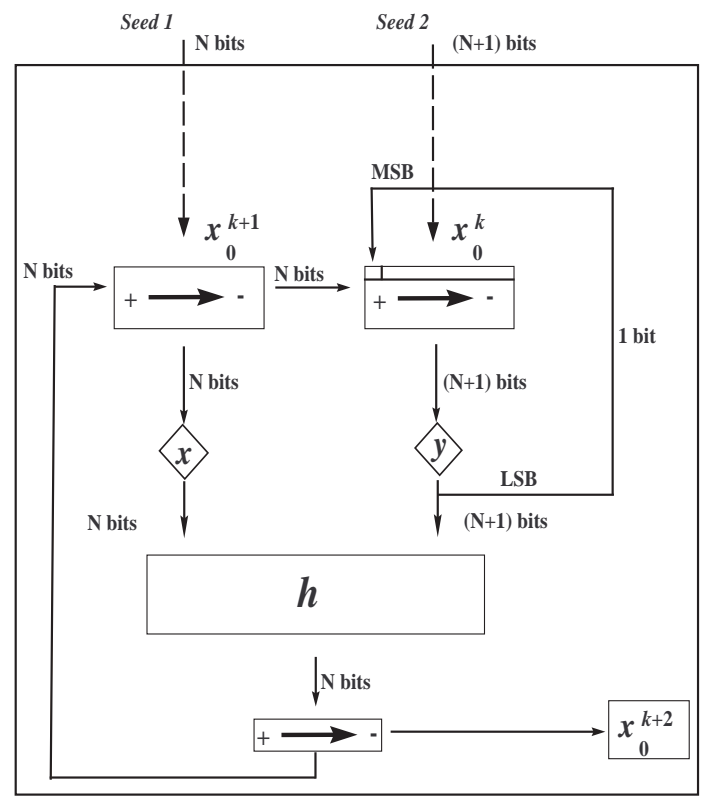

Fig. 1. Basic form of the pseudo-random number generator. MSB and LSB correspond to the most significant bit and the least significant bit, respectively.

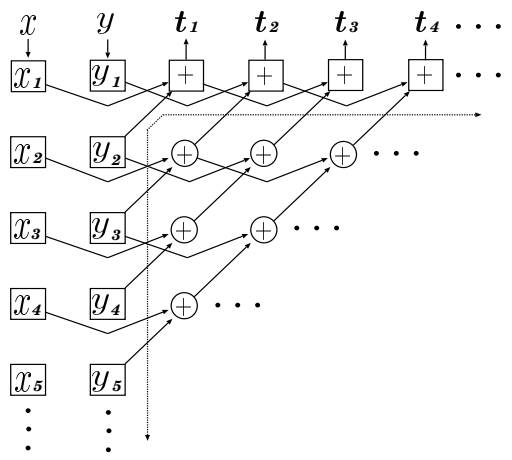

Fig. 2. Generation of a pseudo-random key with input $(\mathbf{x}, \mathbf{y})$ and output $\mathbf{t}=h(\mathbf{x}, \mathbf{y})$. 
November

8

2018

FILE

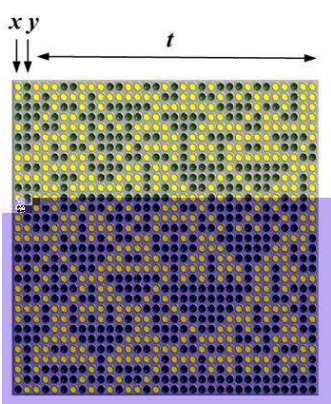

(a)
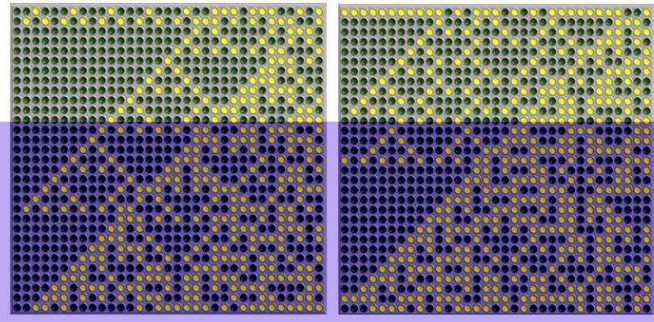

(b)

Fig. 3. Complete backward evolution of the ECA to generate a random sequence of 31 bits with (a) one transformation according to Eq. (3), and with (b) three transformations according to the modified generator given in Eq. (4). We display the sequences $p_{k}, q_{k}$, and $x_{k}$.

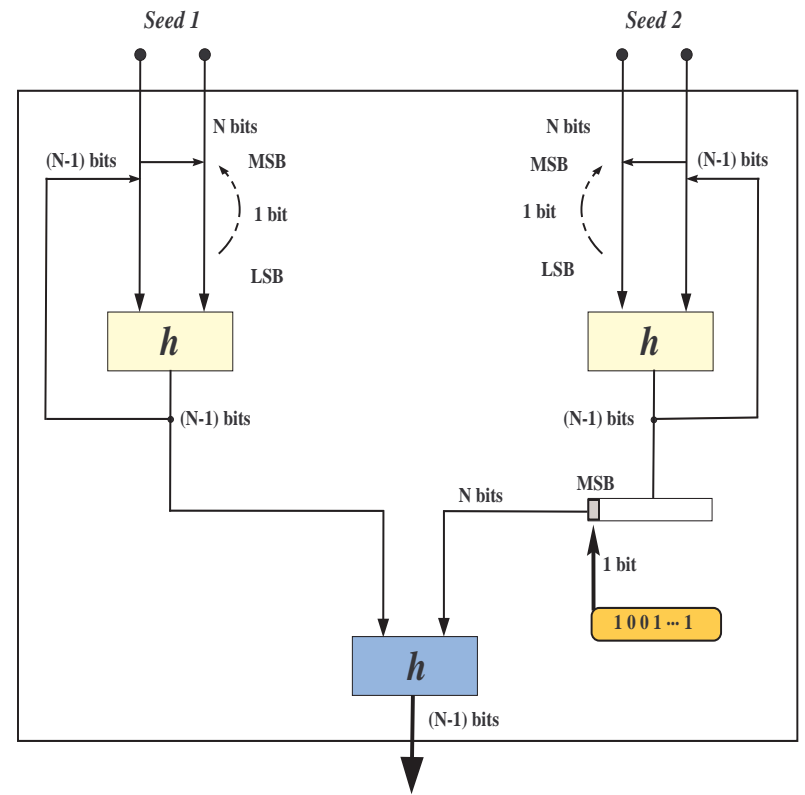

Fig. 4. A generating scheme consisting of three coupled transformations. 
November $\quad 8, \quad 2018 \quad 23: 3 \quad$ WSPC/INSTRUCTION $\quad$ FILE EncripletasvF-IJMPC
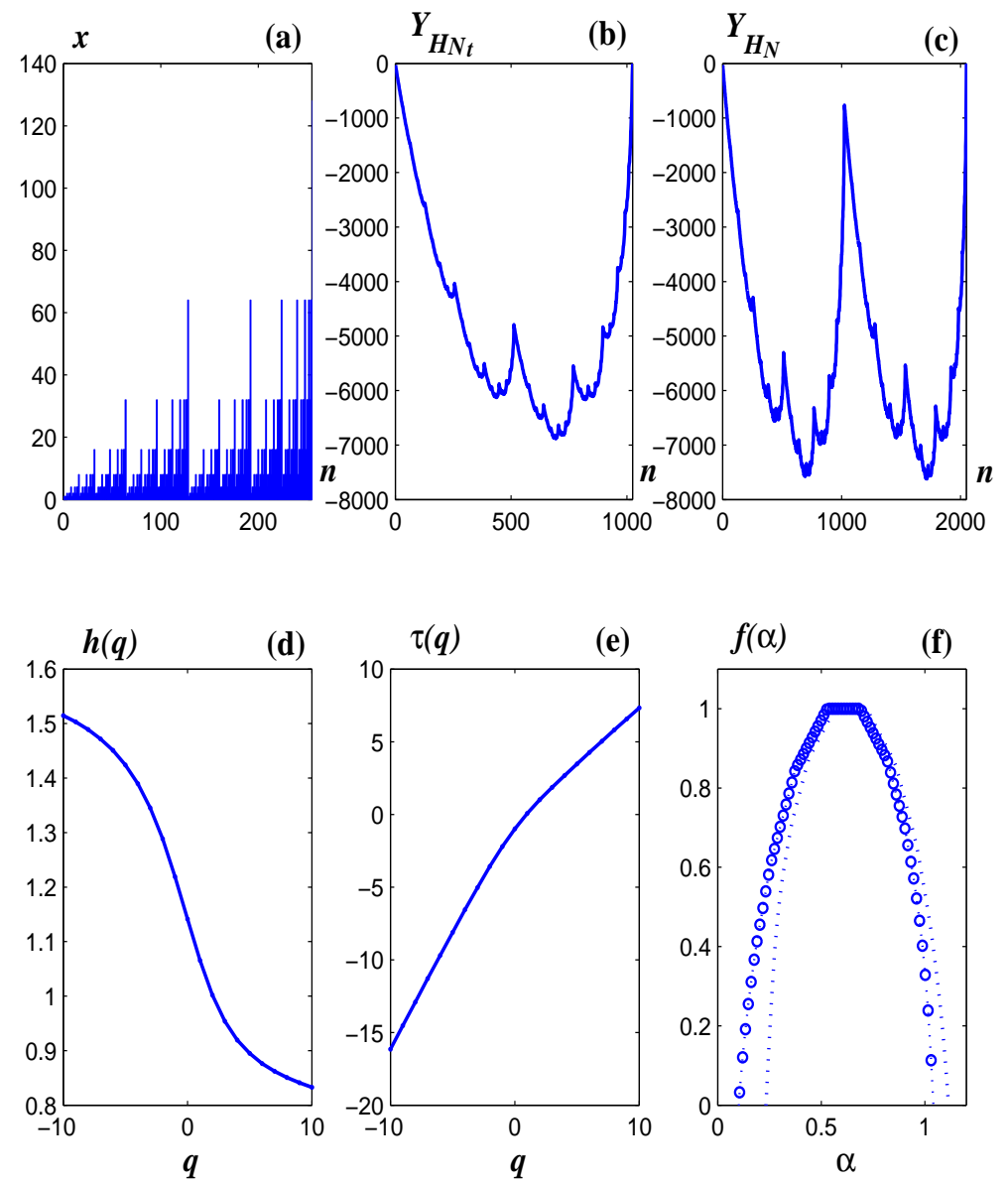

Fig. 5. (a) Time series of the row signal of $H_{1023}$. Only the first $2^{8}$ points are shown of the whole set of $2^{10}-1$ data points. Profiles of the row signal of (b) $H_{N_{t}}$ and (c) $H_{N}$. (d) Generalized Hurst exponent $h(q)$. (e) The $\tau$ exponent, $\tau(q)=q h(q)-1$. (f) The singularity spectrum $f(\alpha)=$ $q \frac{d \tau(q)}{d q}-\tau(q)$. The calculations of the multifractal quantities $h, \tau$, and $f(\alpha)$ are performed with the wavelet-based WMF-DFA. Dotted points correspond to the row signal of $H_{N}$. 
$\begin{array}{llllll}\text { November } & 8 & 2018 & 23: 3 & \text { WSPC/INSTRUCTION } & \text { FILE }\end{array}$ EncripletasvF-IJMPC
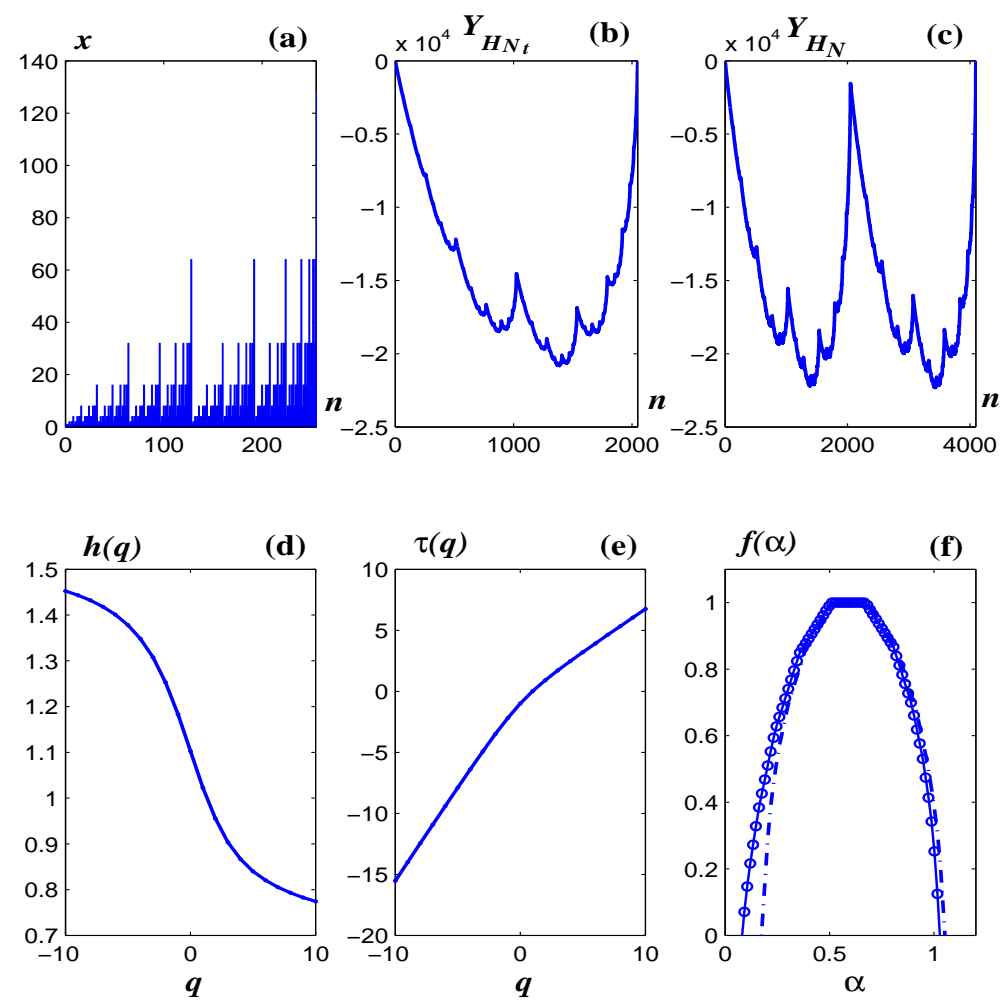

Fig. 6. Same plots as in Fig. 5 but for $H_{2047}$. 
$\begin{array}{llllll}\text { November } & 8, & 2018 & 23: 3 & \text { WSPC/INSTRUCTION } & \text { FILE }\end{array}$ EncripletasvF-IJMPC
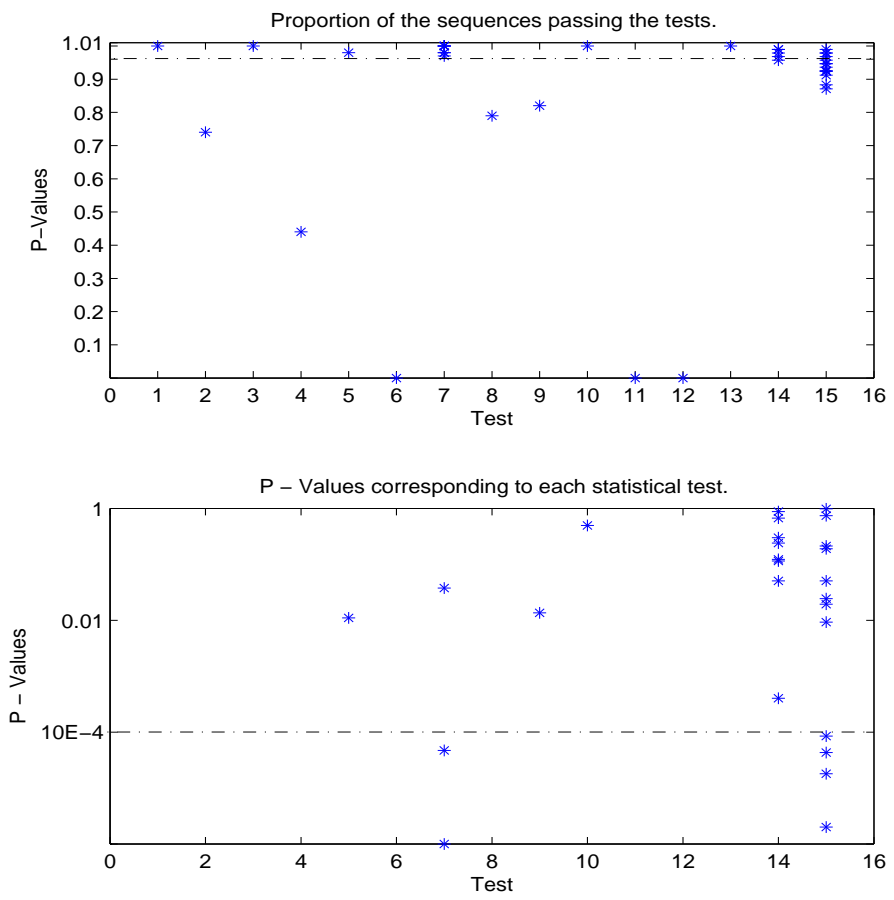

Fig. 7. Proportions and $P$-values $T$ corresponding to $N=7$ bits and one transformation. Dashed line separates the success and failure regions. 
November $\quad 8, \quad 2018 \quad 23: 3 \quad$ WSPC/INSTRUCTION $\quad$ FILE EncripletasvF-IJMPC

Murguía, Mejía Carlos, Rosu \& Flores-Eraña
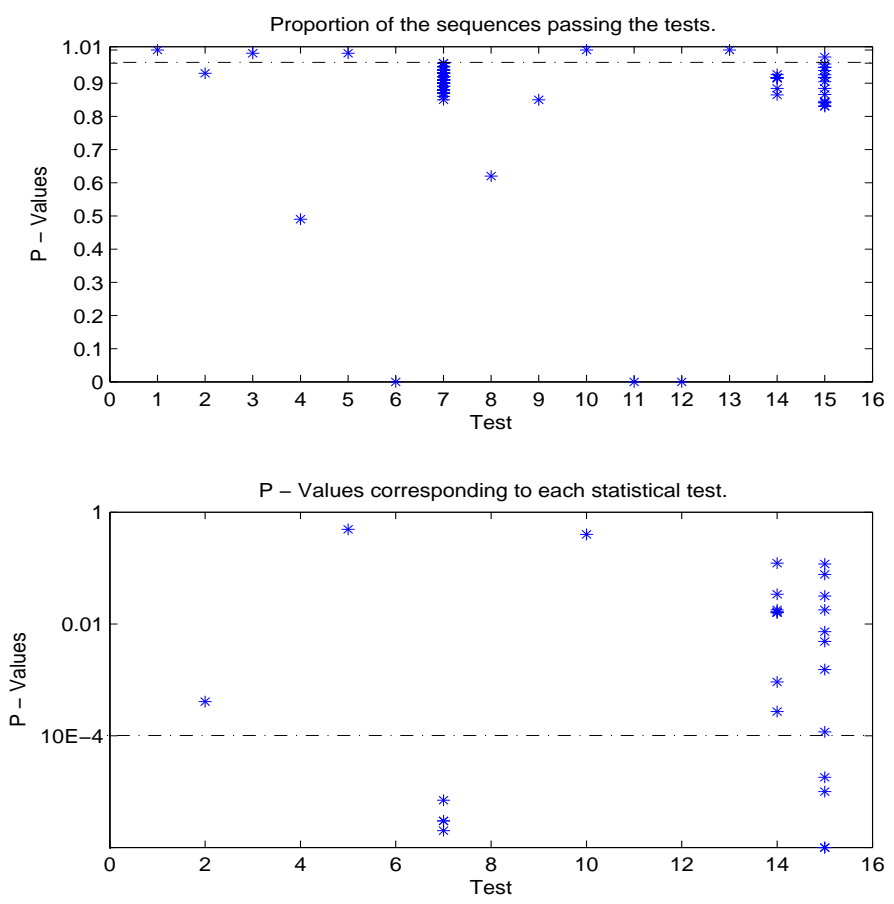

Fig. 8. Proportions and $P-$ values $_{T}$ corresponding to $N=7$ bits and three transformations. Dashed line separates the success and failure regions. 
$\begin{array}{llllll}\text { November } & 8, & 2018 & 23: 3 & \text { WSPC/INSTRUCTION } & \text { FILE }\end{array}$ EncripletasvF-IJMPC
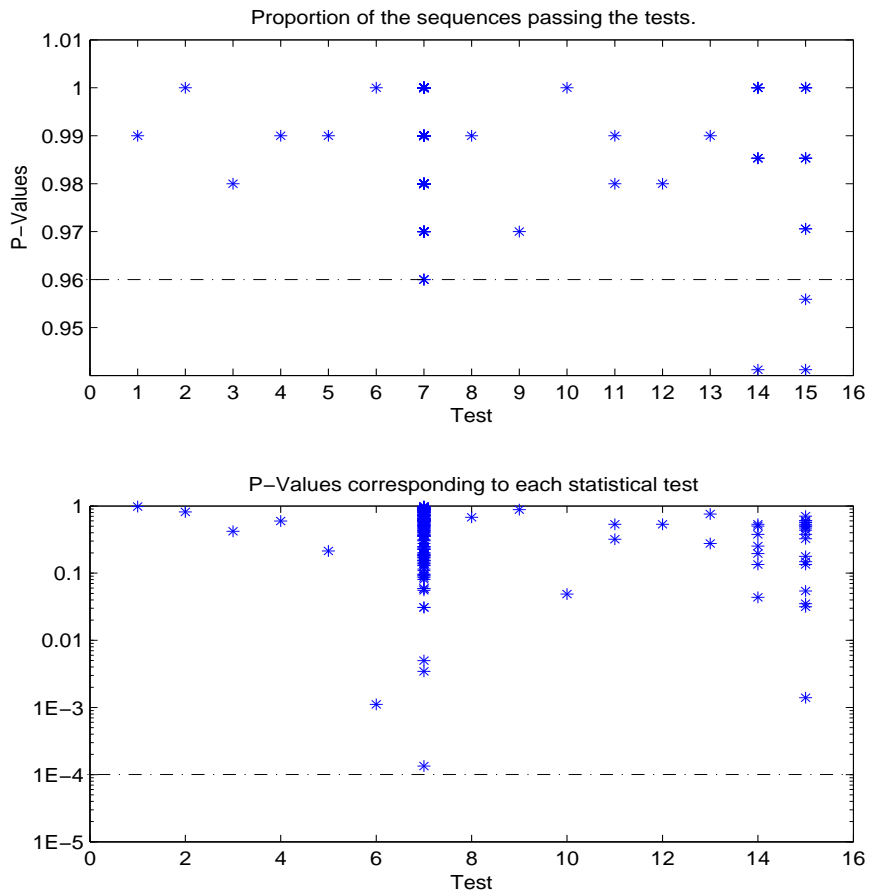

Fig. 9. Proportions and $P-$ values $_{T}$ corresponding to $N=15$ bits and one transformation. Dashed line separates the success and failure regions. 
November $\quad 8, \quad 2018 \quad 23: 3 \quad$ WSPC/INSTRUCTION $\quad$ FILE EncripletasvF-IJMPC
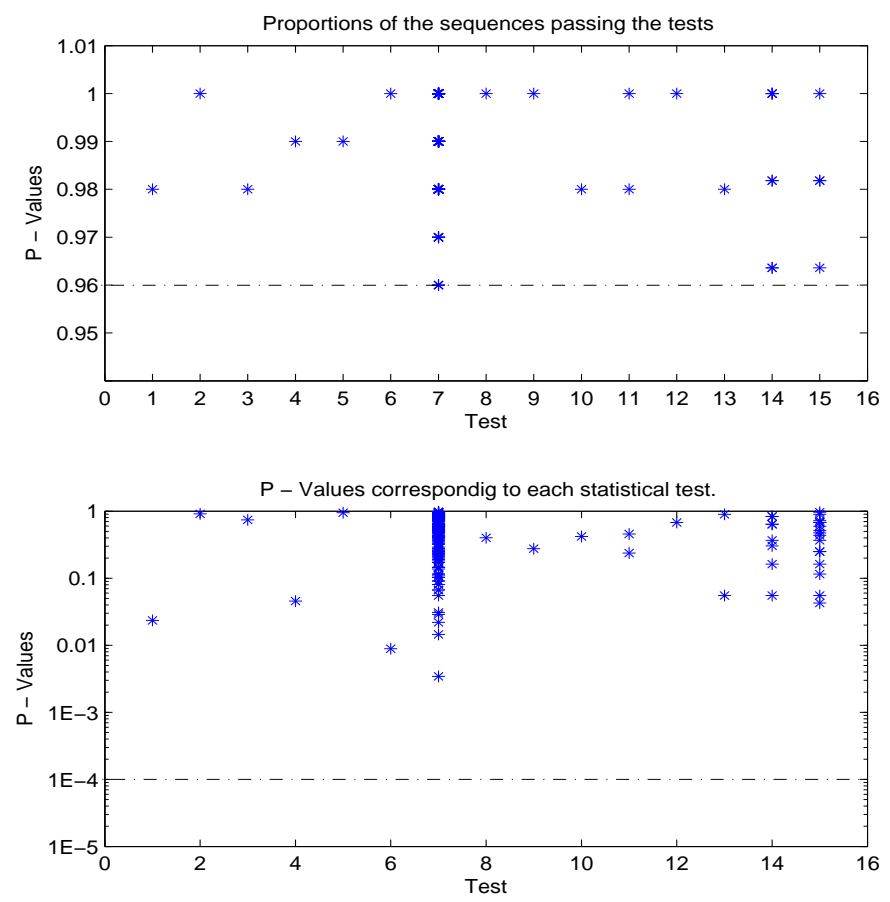

Fig. 10. Proportions and $P-$ values $_{T}$ corresponding to $N=15$ bits and three transformations. Dashed line separates the success and failure regions. 
$\begin{array}{llllll}\text { November } & 8, & 2018 & 23: 3 & \text { WSPC/INSTRUCTION } & \text { FILE }\end{array}$ EncripletasvF-IJMPC

Improvement and Analysis of a Pseudo Random Bit Generator by Means of Cellular Automata
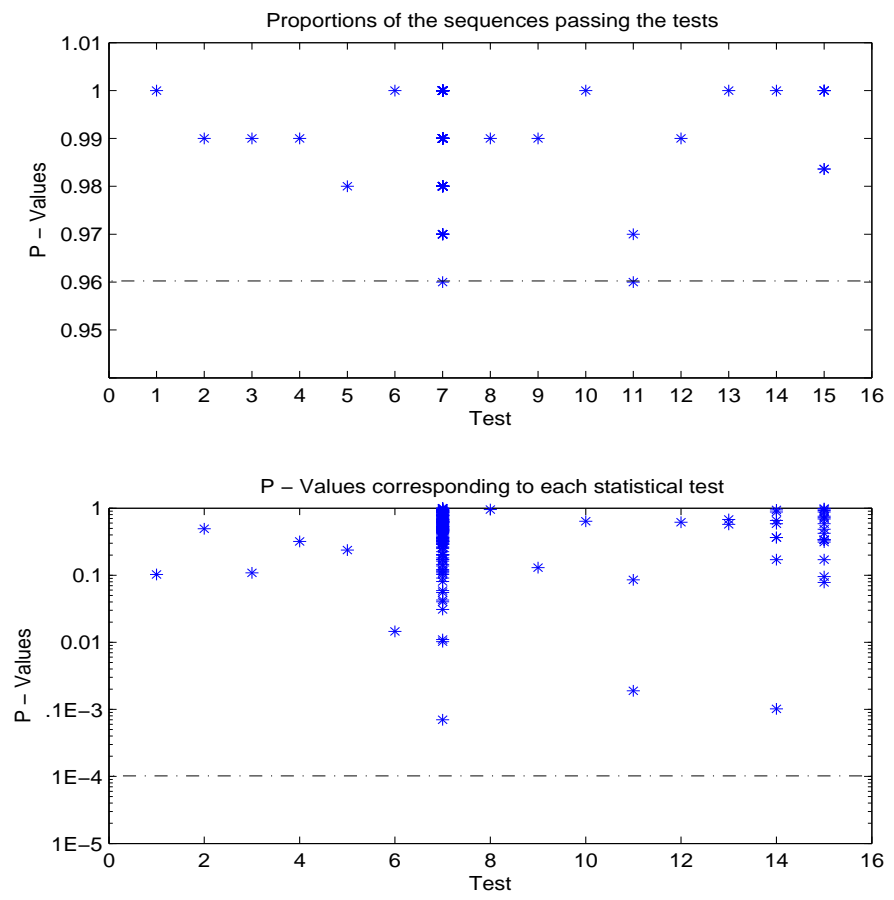

Fig. 11. Proportions and $P$ - values $_{T}$ corresponding to $N=31$ bits and one transformation. Dashed line separates the success and failure regions. 
$\begin{array}{lllll}\text { November } & 8, & 2018 & 23: 3 & \text { WSPC/INSTRUCTION }\end{array}$ EncripletasvF-IJMPC
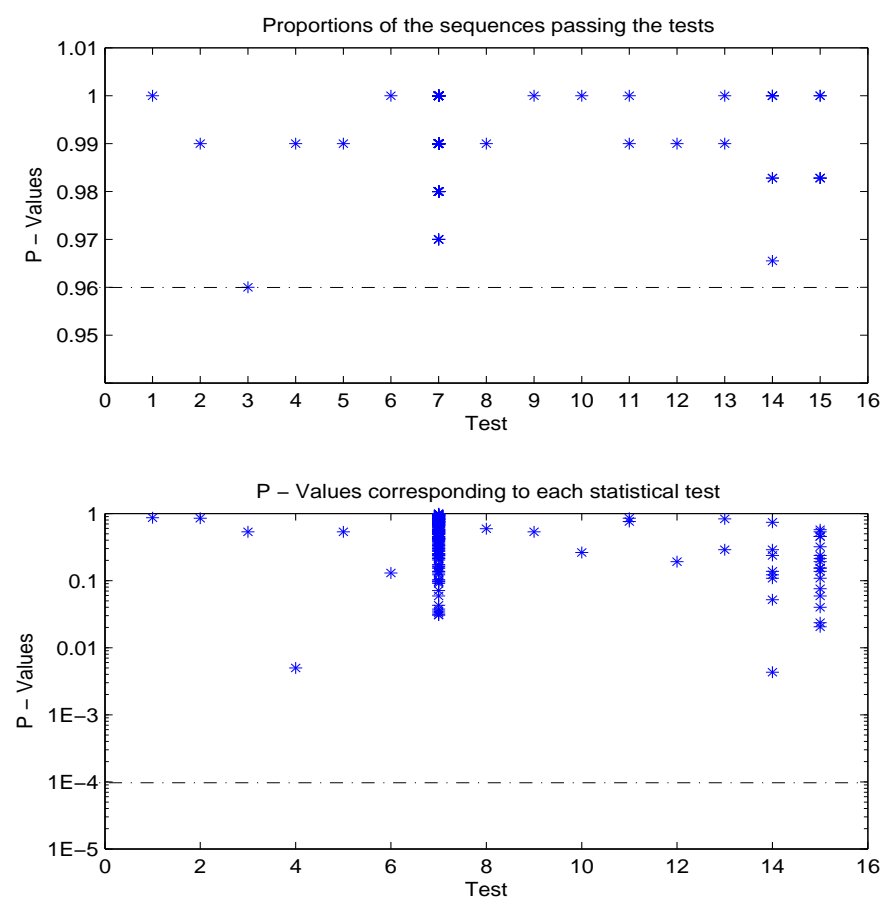

Fig. 12. Proportions and $P-$ values $_{T}$ corresponding to $N=31$ bits and three transformations. Dashed line separates the success and failure regions. 